\title{
MÉTRIQUES PRESCRITES SUR LE BORD DES VARIÉTÉS HYPERBOLIQUES DE DIMENSION 3
}

\author{
FRANÇOIS LABOURIE
}

Une 3-variété hyperbolique compacte $M$ à bord $\partial M$ est dite strictement convexe si deux points quelconques de $M$ peuvent être joints par une géodésique minimisante incluse dans l'intérieur de $M$. Cette condition entraîne que la coubure intrinsèque de $\partial M$ est supérieure à -1 (ici hyperbolique signifie courbure constante -1 ).

Let but de cet article est de démontrer que toutes le métriques sur $\partial M$ à courbure supérieure à -1 sont obtenues ainsi. Plus précisément, nous avons le

Théorème 1. Soit $M$ une variété compacte à bord (différente du tore plein) et qui admette une structure de variété hyperbolique strictement convexe. Soit $g$ une métrique sur $\partial M$ à courbure strictement plus grande que -1 , il existe alors une métrique hyperbolique convexe $h$ sur $M$ qui induise $g$ sur $\partial M$ :

$$
h_{\mid \partial M}=g \text {. }
$$

Le case du tore plein doit être obtenu par d'autres techniques que celles contenues dans cet article.

Ce résultat peut être conçu comme la généralisation de deux théorèmes classiques. Le premier est du à Alexandrov-Pogorelov [9].

Théorème (Alexandrov-Pogorelov). Soit $g$ une métrique sur la sphère $S^{2}$ à courbure strictement supérieure à -1 , il existe alors une immersion isométrique de $\left(S^{2}, g\right)$ dans $H^{3}$, unique aux isométries de $H^{3}$ près. De plus, cette immersion borde un convexe de $H^{3}$.

Ce théorème correspond donc au cas où $M$ est la boule $B^{3}$. Le deuxième théorème, que nous utiliserons dans la démonstration, est le théorème d'uniformisation de Bers qui permet de reconstruire une métrique hyperbolique sur $\operatorname{int}(M)$ à l'aide d'une structure complexe sur $\partial M(\S 2.2)$.

Received January 3, 1991. 
Remarquons que dans ces deux théorèmes la métrique hyperbolique obtenue sur $M$ est unique. Dans nôtre cas, nous sommes incapable de démontrer l'unicité de nôtre métrique $h$, et de répondre ainsi affirmativement à une question de Thurston concernant l'existence et l'unicité d'une telle métrique.

Un autre résultat relié à notre théorème est due à Gromov [4].

Théorème (Gromov). Soit $S$ une surface compacte de genre supérieur ou égal à 2, munie d'une métrique $g$ à courbure supérieure à -1 . Il existe alors un groupe fuchsien $\Gamma$ agissant sur $H^{3}$ telle que $(S, g)$ se plonge isométriquement dans $H^{3} / \Gamma$.

Ce résultat n'est pas a priori un corollaire du nôtre, mais on peut l'obtenir par les mêmes méthodes. Signalons que Gromov ne montre pas non plus l'unicité de $\Gamma$.

\section{Présentation de la démonstration}

Donnons en une idée intuitive. Le point de départ consiste à montrer que l'espace $A$ des solutions des équations de Gauss-Codazzi sur une surface $(S, g)$ est de dimension $6 \gamma-6$, où $\gamma \geq 2$ est le genre de $S$.

Chacune de ces solutions donne naissance à une représentation de $\pi_{1}(S)$ dans $\operatorname{PSL}(2, \mathrm{C})$. De même, si $S=\partial M$, chacune des métriques hyperboliques sur $M$ donne naissance à une représentation de $\pi_{1}(S)$ dans $\operatorname{PSL}(2, \mathbf{C})$. Nous obtenons ainsi dans $\operatorname{Rep}\left(\pi_{1}(S), \operatorname{PSL}(2, \mathbf{C})\right)$ deux sousvariétés, l'une étant l'image de $A$, l'autre l'image de $\mathscr{H}(M)$ l'espace des structures hyperboliques sur $M$.

Une solution à nôtre problème, c'est à dire une variété hyperbolique induisant $g$ sur $S$ est alors un point d'intersection de ces deux sous-variétés. Il nous faut donc démontrer que ce deux sous-variétés s'intersectent.

L'espace $\operatorname{Rep}\left(\pi_{1}(S), \operatorname{PSL}(2, \mathrm{C})\right)$ n'est pas celui que nous allons considérer et c'est l'espace des $\mathbf{C} P^{1}$-structures sur $S$ (localement homémorphe à $\operatorname{Rep}\left(\pi_{1}(S), \operatorname{PSL}(2, \mathbf{C})\right)$ qui va s'avérer utile.

L'existence d'un point d'intersection des deux sous-variétés sera prouvée par un argument homologique.

Plan de l'article:

$\S 2$. Structures hyperboliques sur les variétés de dimension 3: le but de ce section est d'énoncer le théorème d'uniformisation de Bers.

$\S 3$. Immersions isométriques équivalentes: dans ce section, nous étudions la dimension de l'espace des solutions des équations de GaussCodazzi. 
$\S 4$. Surfaces convexes plongées dans les bouts géométriquement finis: ici, nous introduisons des variétés hyperboliques auxiliaires et établissons les estimées géométriques $a$ priori concernant les surfaces convexes qui y sont plongées.

$\S 5 . \mathbf{C} P^{1}$-structures sur une surface compacte: nous montrons comment une solution des équations de Gauss-Godazzi donne naissance à une $\mathbf{C} \boldsymbol{P}^{1}$ structure, de même qu'à une métrique hyperbolique sur $M$ est associée une $\mathbf{C} P^{1}$-structure sur $\partial M$.

$\S 6$. Paramétrisations de l'espace des $\mathbf{C} P^{1}$-structures: il s'agit d'un rappel des résultats de [6] qui relie l'espace des $\mathbf{C} P^{1}$-structures à celui des immersions isomı́triques des surfaces à courbure constante $k$.

$\S 7$. Démonstration du théorème.

\section{Structures hyperboliques sur les variétés de dimension 3}

Ce section est une présentation rapide de résultats élémentaires concernant les variétés hyperboliques, notre but étant d'énoncer le théorème d'uniformisation de Bers, sous la forme qui nous sera le plus utile par la suite. Pour les démonstrations, nous renvoyons à [11] ou à [8].

2.0. Nous nous intéresserons aux variétés hyperboliques non compactes. La classe de variétés qui nous intéresse est celle des variétés convexes cocompactes. Donnons en tout d'abord une définition constructive: si $M$ est une variété hyperbolique à bord convexe, $M$ peut être considérée comme un convexe fermé dans une unique variété hyperbolique complète $\bar{M}$, homotopiquement équivalente à $M$ et que nous appelerons complétée de $M$. Pour construire $\bar{M}$, on peut remarquer par exemple que l'équation de Riccatti (dans la direction extérieure à $\partial M$ ) permet de définir, grâce à l'absence de points focaux, une métrique sans singularités sur $\partial M \times[0, \infty[$ prolongeant celle sur $M$. La variété $\bar{M}$ est alors par définition convexe cocompacte.

D'après cette construction, $\bar{M}$ est homéomorphe à l'intérieur de $M$.

Une autre manière d'arriver à cette définition est de procéder ainsi: soit $\bar{M}=H^{3} / \Gamma$ une 3-variété hyperbolique complète, soit $\Lambda(\Gamma)$ l'ensemble limite de $\Gamma$ dans $\mathbf{C} P^{1}$, le bord à l'infini de $H^{3}$, et $E(\Lambda(\Gamma))$ l'enveloppe convexe de $\Lambda(\Gamma)$ dans $H^{3}$. L'ensemble $E(\Lambda(\Gamma))$ est invariant sous l'action de $\Gamma$, et

$$
N=E(\Lambda(\Gamma)) / \Gamma \subset \bar{M}
$$

est appelée coeur de Nielsen de $\bar{M}$. 
On vérifie aisément que $N$ est aussi l'adhérence de l'intersection de tous les convexes de $\bar{M}$ homotopiquement équivalents à $\bar{M}$.

Par définition $\bar{M}$ est convexe cocompacte si $N$ est compact. Il est facile de vérifier que les deux définitions coïncident.

2.1. A une variété $\bar{M}$ convexe cocompacte on peut associer son bord à l'infini $\partial_{\infty} \bar{M}$, défini comme l'espace des demies-géodésiques divergentes, identifiées si elles sont asymptotes. Si $\mathrm{CP}^{1}$ est le bord à l'infini de $H^{3}$, alors

$$
\partial_{\infty} \bar{M} \approx\left(\mathbf{C} P^{1} \backslash \Lambda(\Gamma)\right) / \Gamma
$$

où $\bar{M}=H^{3} / \Gamma$.

Ce bord à l'infini permet de compactifier $\bar{M}$. En effet, on dira qu'une suite $\left(x_{n}\right)$ de poins de $\bar{M}$ tendant vers l'infini converge vers un point $x_{\infty}$ de $\partial_{\infty} \bar{M}$, si la suite d'arcs géodésiques joignant $x_{n}$ à $\pi\left(x_{n}\right)$, sa projection convexe sur le coeur de Nielsen, converge vers une géodésique asymptote à $x_{\infty}$.

Il est clair que cette compactification de $\bar{M}$ est homéomorphe à tout convexe compact de $\bar{M}$ homotopiquement équivalent à $\bar{M}$ et d'intérieur non vide.

Dans le cas où $\bar{M}$ n'est pas homéomorphe au tore plein, chacune des composantes connexes de $\partial_{\infty} \bar{M}$ est une surface compacte de genre $\gamma$ plus grand ou égal à 2 .

2.2. D'après $(*), \partial_{\infty} \bar{M}$ est muni d'une structure complexe, et le théorème d'uniformisation de Bers nous apprend que cette donnée suffit à reconstruire la métrique. Plus précisément, nous nous donnons $M$ une 3-variété compacte à bord, et nous considérons l'espace $\mathscr{H}(M)$ des métriques hyperboliques complètes sur $\operatorname{int}(M)$, l'intérieur de $M$, telles que l'injection de $\operatorname{int}(M)$ dans $M$, s'étende en une application continue de $\partial_{\infty}(\operatorname{int}(M))$ dans $M$.

Le groupe $\mathscr{G}_{1}$ des difféomorphismes de $M$ dont la restriction à $\partial M$ est isotope à l'identité agit naturellement sur $\mathscr{H}(M)$.

Désignons par Teich $(S)$ l'espace de Teichmüller d'une surface $S$; le théorème d'uniformisation de Bers $[8$, p. 84] s'énonce ainsi:

Théorème (Bers). L'application $\psi_{0}$ de $H(M)=\mathscr{H}(M) / \mathscr{G}_{1}$ dans $\operatorname{Teich}(\partial M)$ qui associe à une métrique hyperbolique sur $\operatorname{int}(M)$, la structure complexe sur $\partial_{\infty} M$ (identifié à $\partial M$ ) est un homéomorphisme, pourvu que $H(M)$ soit non vide.

Remarquons enfin que si $M$ est munie d'une métrique hyperbolique compacte à bord convexe, $H(M)$ est non vide, puisque int $(M)$ est homéomorphe à $\bar{M}$. 


\section{Immersions isométriques équivariantes}

3.0. Soit désormais $S$ une surface connexe compacte orientée de genre supérieur ou égal à 2 et $g$ une métrique riemannienne sur $S$ à courbure strictement plus grande que -1 .

Par définition, une immersion isométrique équivariante de $S$ dans $H^{3}$ est la donnée d'une paire $(f, p)$, où $f$ est une immersion isométrique de $\widetilde{S}$, le revêtement universel de $S$, dans $H^{3}$ et $p$ une représentation de $\pi_{1}(S)$, le groupe fondamental de $S$, dans $\operatorname{PSL}(2, \mathrm{C})$ identifié au groupe des isométries de $H^{3}$, telle que

$$
f(\gamma x)=p(\gamma) f(x),
$$

pour $\gamma$ appartenant à $\pi_{1}(S)$ et $X$ à $\widetilde{S}$.

Le groupe $\operatorname{PSL}(2, \mathrm{C})$ agit à gauche sur $\mathscr{I}(S, g)$, l'espace des immersions isométriques équivariantes, de manière standard

$$
\lambda(f, p)=\left(\lambda \circ f, \lambda p \bar{\lambda}^{1}\right) .
$$

Il est bien connu [10, tome III] que l'espace $I(S, g)=\operatorname{PSL}(2, \mathbf{C}) \backslash \mathscr{I}(S, g)$ (que nous appelerons également espace des immersions équivariantes par abus de langage) s'identifie à l'espace des solutions de l'équation de GaussGodazzi.

Rappelons que, pour une section $\mathrm{B}$, du fibré $S^{2}(B)$ des endomorphismes symétriques de $T S$, ces équations s'écrivent

(i) $\operatorname{det}(B)=\kappa+1$ (équation de Gauss),

(ii) $d^{\nabla}(B)=0$ (équation de Codazzi-Mainardi),

où $\kappa$ désigne la courbure de $S$, et $d^{\nabla}$ la différentielle extérieure covariante associée à la connexion de Levi Civita $\nabla$ de $T S$ :

$$
\begin{aligned}
S^{2}(T S) & \rightarrow \Lambda^{2}(T S) \otimes T S \\
B & \mapsto d^{\nabla} B: d^{\nabla} B(u, v)=\left(\nabla_{u} B\right)(v)-\left(\nabla_{v} B\right)(u) .
\end{aligned}
$$

Nôtre but est de montre tout d'abord:

Lemma 3.1. L'espace $I(S, g)$ est soit vide, soit muni d'une structure de variété sans bord dont la dimension est celle de la dimension $\tau(S)$ de l'espace de Teichmüller de $S$.

Cette structure de variété est celle qui provient de l'identification de $I(S, g)$ avec l'espace des solutions de Gauss-Godazzi.

Preuve. Considérons le sous-fibré $\mathscr{F}^{g}$ de $S^{2}(B)$ dont la fibre $\mathscr{F}_{x}^{g}$ en tout point $x$ de $S$ est constituée des endomorphismes symétriques $B$ de $T_{X} S$ vérifiant

$$
\operatorname{det}(B)=\kappa(X)+1
$$


Pour démontrer nôtre lemme, il nous suffit de démontrer que l'opérateur $d^{\nabla}$ vu comme associant aux sections de $\mathscr{F}^{g}$ des sections de $\Lambda^{2} T S \otimes T S$ a les deux propriétés suivantes.

(i) $d^{\nabla}$ est elliptique.

(ii) Son indice est $\tau(S)$, et son linéarisé à un noyau dont la dimension est $\tau(S)$.

En effet, $I(S, g)$ s'identifiant à l'image inverse de la section nulle par $d^{\nabla}$, nôtre lemme se déduit d'un théorème de fonctions implicites, classique pour les opérateurs elliptiques.

Intéressons nous au linéarisé $L$ de $d^{\nabla}$. Soit tout d'abord $B$ une section de $\mathscr{F}^{g}$. D'après $(*)$ la deux-forme symétrique $\langle B \cdot, \cdot\rangle$ est positive et définit une métrique et en particulier une structure complexe $J$ (la rotation d'angle $\pi / 2$ dans cette métrique).

Cette structure complexe $J$ et $J_{0}$ la structure complexe de $g$ permettent de reconstruire $B$. En effet:

$$
B=-\lambda J_{0} J=G(\lambda J), \quad \text { où } \lambda=\sqrt{\kappa+1} .
$$

En particulier, si $B$ est une section de $\mathscr{F}^{g}$, l'espace tangent en $B$ à l'espace des sections de $\mathscr{F}^{g}$ est identifié par $G$ à l'espace des sections du fibré $Q$ des opérateurs qui commutent à $J$. Le linéarisé $L$ de $d^{\nabla}$ vu de $Q$ dans $\Lambda^{2}(T M) \otimes S^{2} T M$ est alors

$$
\begin{aligned}
L: \Gamma^{\infty}(Q) & \rightarrow \Gamma^{\infty}\left(\Lambda^{2}(S M) \otimes M\right) \\
A & \mapsto-J_{0} d^{\nabla}(A) .
\end{aligned}
$$

Cette interprétation montre que $J_{0} L$ a le même symbole que l'opérateur $\bar{\partial}$ envoyant les différentielles quadratiques sur les champs de vecteurs. En particulier, il est elliptique et son indice est $\tau(S)$.

Pour conclure, il nous faut démontrer que son conoyau est nul. Munissons $Q$ de la métrique

où $d \omega$ est l'élément d'aire de $S$.

$$
\langle\langle A, C\rangle\rangle=\int_{S} \operatorname{tr}(A C) d \omega,
$$

Identifions $\Lambda^{2}(T S) \otimes T S$ à $T S$ grâce à la métrique $g$, et calculons l'adjoint $L^{*}$ de $L$ pour ces métriques: si $\xi \in \Gamma(T S)$ et $A \in \Gamma(G)$

$$
\begin{aligned}
\langle\langle\xi \mid L A\rangle\rangle & =\int_{S}\left\langle\xi \mid-J_{0} d^{\nabla} A\right\rangle d \omega=\int_{S} \operatorname{tr}\left(J_{0} E^{t} A\right) \quad\left(\text { où } E(u)=\nabla_{u} \xi\right) \\
& =\int_{S} \operatorname{tr}\left(J_{0} E J_{0} A J_{0}\right) \quad\left(\operatorname{car}^{t} A=J_{0} A J_{0}\right) \\
& =\int_{S} \frac{1}{2} \operatorname{tr}\left(J\left[J, E J_{0}\right] A\right) .
\end{aligned}
$$


Nous avons donc

$$
\left(L^{*} \xi\right)(u)=-\frac{1}{2}\left(\nabla_{J_{0} u} \xi+J \nabla_{J_{0} J_{u}} \xi\right)
$$

Autrement dit $\xi$ est dans le noyau de $L^{*}$ si et seulement si pour tout $u$ de $T S$ :

$$
J \nabla_{u} \xi=-\nabla_{J_{0} J J_{0} u} \xi
$$

Nous pouvons interpréter cette équation en termes pseudoholomorphes: la connexion $\nabla$ définit une décomposition de l'espace tangent à $T S$ en espace horizonal $H$ et vertical $V$, tous deux identifiés à $T S$. Munissons $V$ de la structure complexe $J$, et $H$ de la structure complexe $-J_{0} J J_{0}$. La variété $T S$ est ainsi munie d'une structure presque complexe, l'équation (**) montre alors que le graphe $\langle\xi\rangle$ de $\xi$ dans $T S$ est une courbe pseudoholomorphe de $T S$. La remarque fondamentale de Lefschetz nous apprend que l'intersection de $\langle\xi\rangle$ avec une autre courbe pseudo-holomorphe (et en particulier le graphe de la section nulle) est strictement positive. On déduit alors de l'hypothése sur la caractéristique d'Euler de $S$ que $\xi$ est constamment nul et conc que le conoyau de $L$ est nul. q.e.d.

De manière analogue, nous démontrons

Lemme 3.2. Soit $\left(g_{t}\right), t$ appartenant à $[0,1]$, une famille différentiable de métriques à courbure strictement plus grande que -1. L'ensemble $W=\bigcup_{t \in[0,1]} I\left(S, g_{t}\right)$ est soit vide, soit muni d'une structure de variété différentiable de dimension $\tau(S)+1$ à bord:

$$
\partial W=I\left(S, g_{0}\right) \cup I\left(S, g_{1}\right) .
$$

Cette structure est celle induite par l'application

$$
\begin{aligned}
W & \rightarrow \Gamma(\operatorname{End}(T S)) \times \mathbf{R} \\
f_{t} & \mapsto\left(\varphi_{t}\left(f_{t}\right), t\right) .
\end{aligned}
$$

Ici $f_{t}$ est une classe d'immersions isométriques équivariantes pour la métrique $g_{t}$, et $\varphi_{t}\left(f_{t}\right)$ est la solution de l'équation de Gauss-Codazzi associée.

Preuve. Raisonnement analogue à la proposition précédente: Il nous faut considérer le fibré

$$
\mathscr{F} \rightarrow(S \times[0,1])
$$

dont la fibre au-dessus d'un point $(s, t)$ est $\mathscr{F}_{x}^{g_{t}}$. On considère alors l'opérateur

$$
d^{\nabla}: \Gamma^{\infty}(\mathscr{F}) \rightarrow \Gamma^{\infty}\left(\Lambda^{2}(T S) \otimes T S\right)
$$


Cet opérateur est Fredholm et nous avons vu que le conoyau du linéarisé était nul et son noyau de dimension $\tau(S)+1$. On conclut à l'aide d'un théorème de fonctions implicites pour les opérateurs Fredholm.

3.3. Remarquons enfin qu'à une surface $S$ immergée dans une variété hyperbolique est naturellement associée un point de $I(S, g)$ (où $g$ est la métrique induite sur $S$ ): soit en considèrant la deuxième forme fondamentale sur $S$, soit plus simplement en considérant l'immersion qui s'en déduit du revêtement universel de $S$ dans $H^{3}$.

Réciproquement à une solution des équations de Codazzi-Mainardi, on peut associer un plongement isométrique de $S$ dans une 3-variété hyperbolique (non complète) et homéomorphe à $S \times[0,1]$ en utilisant un voisinage tubulaire de l'immersion isométrique de $\widetilde{S}$ dans $H^{3}$.

3.4. Si enfin $\Sigma$ n'est pas connexe, on notera $I(\Sigma, g)=\prod_{j \in J} I\left(S_{j, g \mid s_{j}}\right)$, où les $S_{j}, j \in J$, sont les composantes connexes de $S$.

\section{Surfaces convexes plongées dans les bouts géométriquement fini}

4.0. Nous allons introduire des variétés hyperboliques, auxiliaires, les bouts géométriquements finis, et en étudier les surfaces convexes (cf. [5], [6]). Rappelons qu'un bout géométriquement fini est une 3-variété $B$ munie d'une métrique hyperbolique complète telle que:

(i) $B$ est homéomorphe à $S \times[0, \infty[$, où $S$ est une surface compacte de genre plus grand que 2 ,

(ii) la métrique induite sur le bord $B_{0}$ de $B$ est à courbure -1 (le bord $B_{0}$ n'est en général pas lisse),

(iii) le bord de $B$ est développable et convexe, c'est à dire que toute géodésique reliant deux points du bord est tracée sur ce bord (voir Figure 1).

Par construction, le bord d'un bout géométriquement fini est une surface hyperbolique munie d'une lamination géodésique mesurée. Réciproquement, on montre [2] qu'une lamination géodésique mesurée détermine un bout. Un exemple de bout géométriquement fini est obtenu en ôtant à une 3-variété complète et convexe cocompacte son coeur de Nielsen.

Nous avons montré en [5]:

Théorème. Il existe dans chaque bout géométriquement fini une unique surface $S_{k}$ plongée incompressible à courbure constante $k$. De plus les surfaces $S_{k}$, forment un feuilletage de $B$.

Une surface plongée est incompressible si elle sépare un bout géométriquement fini en 2 composantes connexes dont l'un est compacte et contient $B_{0}$. 


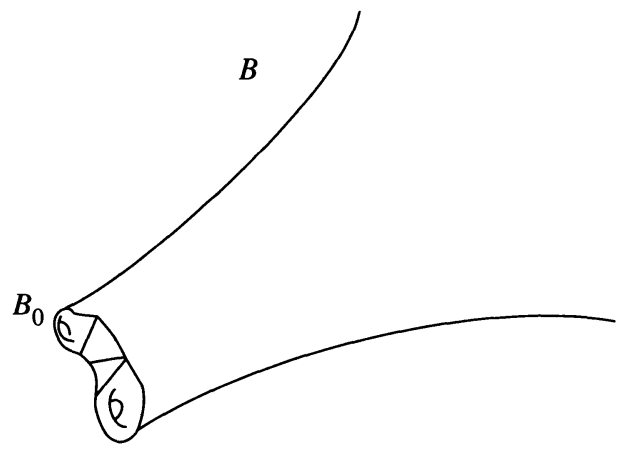

Figure 1

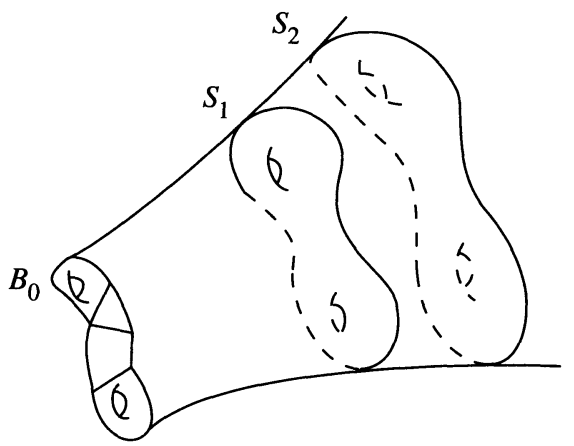

FIGURE 2

4.1. Nous allons nous intéresser maintenant aux surfaces convexes plongées dans un bout $B$. Nous dirons que $S_{2}$ est à l'extérieur de $S_{1}$ si $S_{2}$ et $B_{0}$ sont dans deux composantes connexes distinctes de $B \backslash S_{1}$ (voir Figure 2).

La proposition suivante contient les estimées a priori concernant les surfaces convexes plongées dans les bouts et qui nous seront utiles lors de la démonstration du théorème.

Proposition 4.1. Soit $S$ une surface plongée convexe et incompressible dans un bout $B$. (i) Si $K$ est la fonction courbure de $S$ et si $k \leq$ $\inf _{x \in S}(K(X))$, alors $S$ est à l'extérieur de $S_{k}$.

(ii) $S i S_{k}$ est à l'extérieur de $S$, alors

$$
k \geq \frac{2 \pi \chi(S)}{\operatorname{Aire}(S)},
$$

de même si $S$ est à l'extérieur de $S_{k}$, alors 


$$
k \leq \frac{2 \pi \chi(S)}{\operatorname{Aire}(S)} .
$$

(iii) Si s appartient à $S$, alors' $C_{1} \leq d\left(s, B_{0}\right) \leq C_{2}$, où $C_{i}$ ne dépend que de $B$ et de la métrique induite de $S$.

(iv) Soit $H$ la courbure moyenne de $S$, alors si do est l'élément d'aire de $S, \int H d \sigma \leq C_{3}$, où $C_{3}$ ne dépend que de $B$ et de la métrique sur $S$.

Démonstration. (i) Les surfaces $S_{k}$ feuillettent $B$. Soit donc $k_{0}$ le plus grand des $k$ tel que $S$ est à l'extérieur de $S_{k}$. En particulier, il existe un point $s_{0}$ de $S$ en lequel $S_{k_{0}}$ est tangente intérieurement. Il est par ailleurs aisé de voir que si deux surfaces convexes $S_{1}$ et $S_{2}$ sont telles que $S_{1}$ est tangente intérieurement à $S_{2}$ en $s$, alors la courbure en $s$ de $S_{1}$ est plus grande que la courbure en $s$ de $S_{2}$.

(ii) Comme $B$ est à courbure négative, la projection convexe (cf. [1]) de $S_{k}$ sur $S$ est contractante et les inégalités se déduisent d'inégalités sur les aires.

(iii) D'après (i), on en déduit que $d\left(S, B_{0}\right) \geq d\left(S_{k}, B_{0}\right)$ pour $k=$ $\inf K$, ce qui donne notre première inégaliteé. Ensuite d'après (ii) la surface $S_{k i} k=2 \pi \chi(S) / \operatorname{Aire}(S)$, rencontre $S$. Dès lors

$$
d\left(s, B_{0}\right) \leq \operatorname{Diam}(S)+\sup _{x \in S_{k}}\left(d\left(x_{1} B_{0}\right)\right) .
$$

(iv) D'après (iii) il existe $\varepsilon$ ne dépendant que de $g$ (la métrique induite sur $S$ ) et $B$ tel que le rayon d'injectivité de $B$ en tout point de $S$ est supérieur à $\varepsilon$. Nous pouvons donc recouvrir $S$ (dans $B$ ) par $N$ (ne dépendant que de $g, B$, et $\varepsilon$ ) boules de rayon $\varepsilon$ dans $B$ (et donc homéomorphes à des boules) centrées sur des points de $S$.

Nous avons par ailleurs montré (cf. 5.4(iii) de [7]) que si $S$ était une surface incluse dans le bord d'un convexe contenu dans la boule de rayon $\varepsilon$ centrée sur un point de $S$, il existait une constante $C$ ne dépendant que de $\varepsilon$ et de la borne inférieure de la courbure de $S$ tel que l'intégrale de la courbure moyenne sur $S$ était bornée par $C$. On en déduit aisément (iv).

\section{5. $\mathbf{C} P^{1}$-structures sur une surface compacte}

5.0. Soit $S$ une surface connexe compacte, orientée, de genre $\gamma \geq 2$ et sans bord. Une $\mathbf{C} P^{1}$-structure sur $S$ est la donnée d'une paire $(g, p)$, où $g$ est une immersion de $\widetilde{S}$, le revêtement universel de $S$, dans $\mathbf{C} P^{1}$, et $p$ une représentation de $\pi_{1}(S)$ le groupe fondamental de $S$ dans 
$\operatorname{PSL}(2, \mathbf{C})$, le groupe des transformations projectives complexes de $\mathbf{C} P^{1}$ telle que

$$
g(\gamma x)=p(\gamma) g(x)
$$

pour tout $\gamma$ de $\pi_{1}(S)$ et $x$ de $S$.

Si $\mathscr{G}_{0}$ est le groupe des difféomorphismes de $S$ isotopes à l'identité, $\mathscr{G}_{0} \times \operatorname{PSL}(2, \mathbf{C})$ agit sur l'espace $\mathscr{C} \mathscr{P P}^{1}(S)$ des $\mathbf{C} P^{1}$-structures de $S$ de manière standard:

$$
\left(g_{0}, \lambda\right)(f, p)=\left(\lambda f_{0} g_{0}, \lambda p \lambda^{-1}\right)
$$

Par abus de langage, nous appelerons l'espace

$$
\mathbf{C} P^{1}(S)=\mathscr{C P P}^{1}(S) / \mathscr{G}_{0} \times \operatorname{PSL}(2, \mathbf{C})
$$

espace des $\mathbf{C} P^{1}$-structures de $S$ et chacun de ses éléments, $\mathbf{C} P^{1}$-structure.

Si $\Sigma$ est non connexe, on notera

$$
\mathbf{C} P^{1}(\Sigma)=\prod_{j \in J} \mathbf{C} P^{1}\left(S^{j}\right),
$$

où les $S^{j}$ sont les composantes connexes de $\Sigma$.

Si $S$ est connexe, il est classique de montrer [3] que $\mathbf{C} P^{1}(S)$ est une boule de dimension $12 \gamma-12$ si le genre $\gamma$ de $S$ est supérieur ou égal à 2.

5.1. Exemples. (i) Une immersion isométrique équivariante de $(S, g)$ dans $\mathbf{H}^{3}$ si $K>-1$, définit naturellement une $\mathbf{C} P^{1}$-structure sur $S$. En effet, soit $(f, p)$ Une immersion isométrique équivariante de $(S, g)$ dans $\mathbf{H}^{3}$, l'application de Gauss hyperbolique $\gamma$ qui associe à un point de $f(\widetilde{S})$, le point du bord à l'infini de $\mathbf{H}^{3}$ (identifié à $\mathbf{C} P^{1}$ avec sa structure projective) définit par la normale extérieure, est une immersion puisque $f(\widetilde{S})$ est convexe (voir Figure 3).

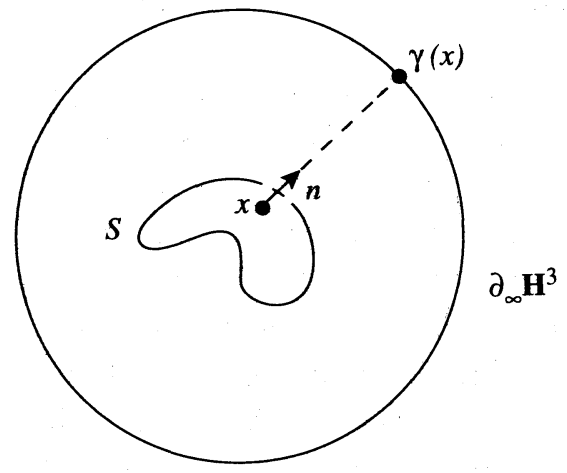

FIGURE 3 


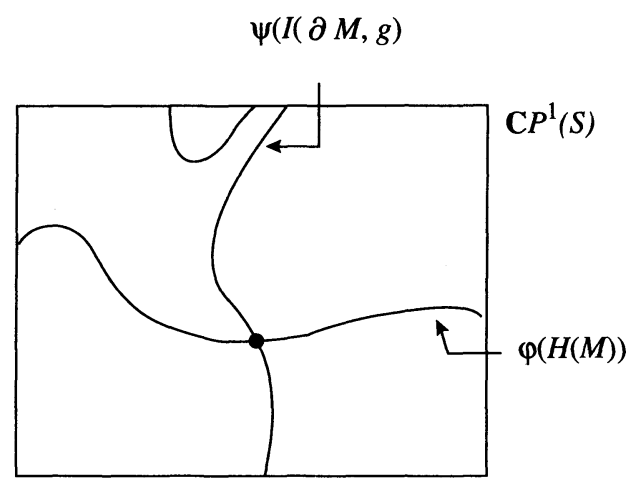

FIGURE 4

Il est alors clair que $(\gamma \circ f, p)$ est une $\mathbf{C} P^{1}$-structure sur $S$ dite $\mathbf{C} P^{1}$ structure associée. Plus précisément, nous avons

Proposition 5.1(i). Soit $g$ une métrique à courbure strictement plus grande que -1 sur $S$, l'application $\psi$ de $I(S, g)$ dans $\mathbf{C} P^{1}(S)$ envoyant une immersion isométrique équivariante sur sa $\mathbf{C} P^{1}$-structure associée est continue.

De même, si $g_{t}$ est une famille continue de métriques à courbure $>-1$ et $W$ la variété construite en $3.2, \psi$ de $W$ dans $\mathbf{C} P^{1}(S)$ est continue.

Proof. Immédiat.

(ii) Si maintenant $\bar{M}$ est une variété hyperbolique convexe cocompacte, nous avons vu $\S 2.1$ que si $\bar{M}=\mathbf{H}^{3} / \Gamma$ nous avions

$$
\partial_{\infty} \bar{M} \approx\left(\mathbf{C} P^{1} \backslash \Lambda(\Gamma)\right) / \Gamma .
$$

En particulier, chaque composante connexe de $\partial_{\infty} \bar{M}$ est munie d'une $C P^{1}$-structure canonique. Nous avons alors la proposition, analogue de 5.1(i)

Proposition 5.1(ii). Soit $M$ une variété compacte à bord $\partial M$ l'application $\varphi$ de $H(M)$ dans $\mathbf{C} P^{1}(\partial M)$ qui associe à une métrique hyperbolique sur $\operatorname{int}(M)$ la $\mathbf{C} P^{1}$-structure de son bod à l'infini est continue.

5.2. Soit $M$ une 3-variété hyperbolique compacte à bord convexe. La $\mathbf{C} P^{1}$-structure sur $\partial M$ induite de la structure hyperbolique sur le complété $\bar{M}$ de $M$, coïncide bien évidemment avec la $\mathbf{C} P^{1}$-structure associée à l'immersion isométrique de $(\partial M, g)$ ( $g$ est la restriction de la métrique hyperbolique sur $M$ ) dans $\bar{M}$. Autrement dit cette $\mathbf{C} P^{1}$-structure est un point d'intersection dans $\mathbf{C} P^{1}(S)$ de $\varphi(H(M)$ ) et $\psi(I(\partial M, g)$ ) (voir Figure 4). 
5.3. Un dernier et important exemple est la $\mathbf{C} P^{1}$-structure sur le bord à l'infini d'un bout géométriquement fini, structure qui s'obtient comme pour les variétés complètes. Un théorème de Thurston (non publié) nous dit que les $\mathbf{C} P^{1}$-structure sont en bijection naturelle avec les bouts géométriquement finis.

\section{Paramétrisations de l'espace des $\mathbf{C} P^{1}$-structures}

6.1. Nous allons dans cette section rappeler les résultats obtenus en ([5], [6]). Nous avons démontré le résultat suivant sur les paramétrisations de $\mathbf{C} P^{1}(S)$.

Théorème (théorème $1 \mathrm{de}[6]$ ). Soit $k$ un nombre appartenant à ]-1, 0[, et $\tau$ une $\mathbf{C} P^{1}$-structure sur $S$, il existe alors une unique métrique $g_{k}$ courbure constante $k$ sur $S$ et une unique immersion isométrique équivariante $(f, p)$ de $\left(S, g_{k}\right)$ dans $H^{3}$ telle que $\tau$ soit associée à $(f, p)$.

Nous avons ainsi construit une application continue $\pi_{k}$ de $\mathbf{C} P^{1}(S)$ dans Teich $(S)$ associant à $\tau$ la structure complexe de $g_{k}$.

Par construction, la fibre de $\pi_{k}$ est $I\left(S, g_{k}\right)$. Nous avons de plus démontré que $I\left(S, g_{k}\right)$ était homéomorphe à $\mathbf{R}^{\tau(S)}$.

6.2. Par ailleurs, si $M$ est une 3-variété hyperbolique complète convexe cocompacte et $N$ son coeur de Nielsen, nous avons démontré (théorème 2 de [5]) que $M \backslash N$ était feuilletee par des surfaces à courbure constante $k, k \in[-1,0$ [, et done homéomorphes à $\partial M$ (elles bordent des convexes). Ceci définit une application $f_{k}$ de $H(M)$ dans Teich $(\partial M)$ associant à une métrique hyperbolique sur $\operatorname{int}(M)$, la structure complexe définie par la surface à courbure constante $k$ dans int $(M)$ du théorème 2 de [5]. Par construction

$$
f_{k}=\pi_{k} \circ \varphi
$$

Enfin, nous avons montré

Proposition 6.2 (Lemma 4.5 de [5]). L'application $f_{k}$ est propre et de degré 1.

Sans la redémontrer, donnes l'idée de cette proposition: elle consiste à montrer que quand $k$ tend vers $0, f_{k}$ est proprement homotope à $f_{0}=\pi_{0} \circ \varphi$, où $\pi_{0}$ est l'application de $\mathbf{C} P^{1}(S) \rightarrow$ Teich $(S)$ associant à une $\mathbf{C} P^{1}$-structure, la structure conforme induite par la développante. Maintenant le théorème de Bers nous dit justement que $f_{0}$ est un homéomorphisme.

Autrement dit cela revient à rendre homologue les fibres $I\left(S, g_{k}\right)$ 
de $\pi_{k}$ avec celle de $\pi_{0}$, fibres que l'on considère en quelque sorte comme l'espace des immersions isométriques équivariantes de $S$ muni d'une "métrique plate d'aire infinie et conforme à une structure complexe donnée."

6.3. Remarquons pour conclure que si deux bouts géométriquement finis $B_{1}$ et $B_{2}$ définissent la même $C P^{1}$-structure, ces deux bouts sont isométriques. En effet, $B_{1}$ et $B_{2}$ sont feuilletées par des surfaces $S_{k}^{1}$ et $S_{k}^{2}$ à courbure constante $k$. D'après $\S 6.1, S_{k}^{1}$ et $S_{k}^{2}$ sont isométriques et leurs plongements dans $B_{1}$ et $B_{2}$ induisent la même deuxième forme fondamentale. On en déduit aisément que leurs voisinages tubulaires et finalement $B_{1}$ et $B_{2}$, sont isométriques. Ceci est aussi un corollaire des résultats de Thurston ( $(5.3)$.

\section{Démonstration du théorème}

7.0. L'idée de base est la suivante. Soit $M$ une variété compacte à bord admettant une structure hyperbolique convexe et différente du tore plein. Chacune des composantes connexes de $\partial M$ est alors une surface compacte de genre supérieure ou égal à 2 .

Soit $g$ une métrique sur $\partial M$ à courbure strictement supérieur à -1 . Dans $C P^{1}(\partial M)$ vivent alors deux sous-variétées, l'image de $H(M)$ et celle de $I(\partial M, g)$ (cf. 5.1(i) et (ii)). Il nous faut (et en fait cela suffira) démontrer que ces deux sous-variétés, de dimension moitié de celle de $\mathbf{C P} P^{1}(\partial M)$, s'intersectent.

Pour cela, nous allons déformer $g$ en une métrique à courbure constante $g_{k}$ et utiliser le fait que $I\left(\partial M, g_{k}\right)$ et $H(M)$ s'intersectent ( $(5.2)$ et montrer par des arguments homologiques que l'intersection persiste lors de la déformation.

L'un des points cruciaux de la démonstration est alors de démontrer la propreté de certaines applications (cf. 7.0). Plus précisément, soit $g_{0}$ une métrique sur $\partial M$ à courbure $>-1 g_{t}, t \in[0,1]$, une famille différentiable de métriques et $k$ un nombre réel telles que

(i) $g_{t}$ soit à courbure $\geq+k>-1$,

(ii) $g_{1}$ soit à courbure constante $k$.

Une telle famille de métriques existe puisque (cf. [4, P. 289]) l'ensemble des métriques à courbure plus grande que -1 sur une surface est connexe.

Construisons, comme en 3.2 , la variété

$$
W=\bigcup_{t} I\left(S, g_{t}\right)
$$

La démonstration du théorème, dans le cas où $M$ est différente du tore 
plein, se fait en deux temps: D'abord nous démontrons la proposition 7.0, puis montrerons (c'est l'argument d'homologie) qu'elle entraîne le théorème.

Proposition 7.0. Soit $\psi$ l'application continue (cf. 4.1(i)) de $W$ dans $\mathbf{C} P^{1}(\partial M)$ envoyant une immersion isométrique équivariante sur sa $\mathbf{C} P^{1}$. structure associée alors:

(i) $\psi$ est propre de $W_{0}$ dans $\mathbf{C} P^{1}(S)$,

(ii) $\pi_{k}\left(\psi\left(W_{0}\right)\right)$ est relativement compacte dans l'espace de Teichmüller. Ici $W_{0}$ désigne la composante connexe de $I\left(S, g_{k}\right)$ dans $W$.

Nous allons avoir besoin d'un lemme:

Lemma 7.1. Soit $(f, p)$ une immersion isométrique équivariante de $\left(S, g_{t}\right)$ appartenant $\grave{a} W_{0}$, alors $(f, p)$ provient d'un plongement isométrique incompressible dans un bout géométriquement fini $B$ (au sens de §3.3).

Démonstration. Soit $V$ l'ensemble des immersions isométriques équivariantes de $W$ qui proviennent au sens se $\S 3.3$ d'un plongement isométrique incompressible dans un bout géométriquement fini $B$. Montrons le résultat par connexité.

(a) Montrons que $V$ est fermé. Soit en effet $\left(g_{n}\right)$ une suite de métriques convergeant vers $\left(g_{\infty}\right),\left(B_{n}\right)$ une suite de bouts géométriquements finis et $\left(f_{n}\right)$ une suite de plongements isométriques incompressibles de $\left(S, g_{t_{n}}\right)$ dans $B_{n}$ telle que la suite des deuxièmes formes fondamentales des $f_{n}$ converge.

La suite des $\mathbf{C} P^{1}$-structures associées aux $f_{n}$ converge, ce qui entraîne (l'espace des $\mathbf{C} P^{1}$-structures est homéomorphe à l'espace des bouts géométriquement finis) que la suite de bouts $\left(B_{n}\right)$ converge vers un bout $B_{\infty}$. D'après 4.1 (iii) et le théorème d'Ascoli, la suite $\left(f_{n}\right)$ va converger $C^{0}$ vers une application $f_{\infty}$ de $S$ dans $B_{\infty}$. Comme la suite des deuxièmes formes fondamentales converge, $f_{\infty}$ est $C^{\infty}$ et sa deuxième forme fondamentale est la limite des deuxièmes formes fondamentales.

(b) Montrons que $V$ est ouvert. Soit $f_{1}$ de $\left(S, g_{1}\right)$ dans $B_{1}$ un bout géométriquement fini on peut alors trouver un voisinage $U$ de $B_{1}$ dans l'espace des bouts, tel qu'à tout bout $B$ de $U$ soit associée continuement une immersion isométrique $f_{B}$ de $\left(S, g_{B}\right)$ dans $B$, où $g_{B_{1}}=g_{1}$ et $f_{B+1}=f_{B}$.

Si maintenant $(f, p)$ est une immersion isométrique équivariante $\varepsilon$ proche de $f_{1}$, il existe un bout $B_{2}$ proche de $B_{1}$ dont $p$ soit la représentation d'holonomie.

Par ailleurs si $\left(f^{\prime}, p\right)$ est une immersion isométrique équivariante de 
$\left(S, g^{\prime}\right)$ et si $\left(f^{\prime \prime}, p\right)$ est une immersion isométrique équivariante de $\left(S, g^{\prime \prime}\right)$ suffisamment proche ayant la même représentation d'holonomie, alors $\left(f^{\prime \prime}, p\right)$ provient d'un plongement dans le voisinage tubulaire de $\left(f^{\prime}, p^{\prime}\right)$.

En particulier, $(f, p)$ provient d'un plongement dans le voisinage tubulaire de $f_{B_{2}}(S)$ et donc d'un plongement dans un bout.

7.2. Preuve de la proposition 7.0. Montrons (i), soit donc $\left(f_{n}\right)$ une suite de plongements isométriques incompressibles de $\left(S, g_{t_{n}}\right)$ dans une suite convergeante de bouts géométriquement finis $\left(B_{n}\right)$. On peut supposer que $\left(g_{t_{n}}\right)$ onverge. D'après 4.1(iii) et le théorème d'Ascoli, la suite $\left(f_{n}\right)$ converge $C^{0}$ vers une application $f_{\infty}$ de $\left(S, g_{t_{\infty}}\right)$ dans $B_{\infty}$. D'après 4.1(iv) et le théorème $\mathrm{D}$ de [7], la convergence est $C^{\infty}$ ce qui montre la propreté.

Montrons (ii), soit $\left(f_{t}\right)$ de $\left(S, g_{t}\right)$ dans un bout $B$ un plongement isométrique équivariant représentant un élément de $W_{0}$. D'après 4.1(i), $f_{t}(S)$ est à l'extérieur de la surface $S_{k}$ a courbure constante $k$ dans le bout $B$.

En particulier, comme la projection sur un convexe est contractante, le diamètre de $S_{k}$ est borné. Ceci montre que $\pi_{k}\left(\psi\left(W_{0}\right)\right)$ est inclus dans l'ensemble des surfaces à courbure constante $k$, dont le diamètre est majoré par la borne supérieure du diamètre des surfaces $\left(S, g_{t}\right)$. Or ce dernier ensemble est compact.

7.3. Montrons que la proposition 7.0 entraîne le théorème. Nous savons que $\partial W_{0}=I\left(S, g_{1}\right) \cup \widetilde{I}\left(S, g_{0}\right)$, où $\widetilde{I}\left(S, g_{0}\right)$ est inclus dans $I\left(S, g_{0}\right)$. Il nous suffit de montrer que $\psi\left(\widetilde{I}\left(S, g_{0}\right)\right)$ intersecte $\varphi(\mathscr{H}(M))$. En effet, si $\varphi((f, p))=\psi(M, h)$ est un point d'intersection, soit $N$ le coeur de Nielsen de $(M, h)$.

La variété $M \backslash N$ est une union finie de bouts géométriquement finis $\bigcup_{i} B_{i}$. D'après $\S 3.3,(f, p)$ provient alors d'un plongement incompressible de $\left(S, g_{0}\right)$ dans $\bigcup_{i} B_{i}$. En particulier, $\left(S, g_{0}\right)$ se plonge isométriquement dans $(M, h)$ comme le bord d'un convexe homotopiquement équivalent à $M$.

Montrons donc que $\psi\left(\widetilde{I}\left(S, g_{0}\right)\right)$ et $\varphi(\mathscr{H}(M))$ s'intersectent. Nous savons que $\mathbf{C} P^{1}(S)$ est homéomorphe à $\mathbf{R}^{6 g-6} \times \mathbf{R}^{6 g-6}, \pi_{k}$ étant alors donné par la projection sur le premier facteur. D'après $\S 2.2, H(M)$ est une variété de dimension $6 g-6$, et d'après $\S 3.2 W_{0}$ est de dimension $6 g-5$. Nôtre résultat découle alors de $\S \S 6.2,7.0$, et du lemme suivant:

Lemma. Soit $M$ une variété sans bord de dimension $n, N$ une variété à bord de dimension $n+1$, telle que $\partial N=N_{1} \cup N_{2}$, où $N_{1}$ et $N_{2}$ sont deux variétés sans bord de dimension $n$. Considérons $p_{1}$ la projection de 


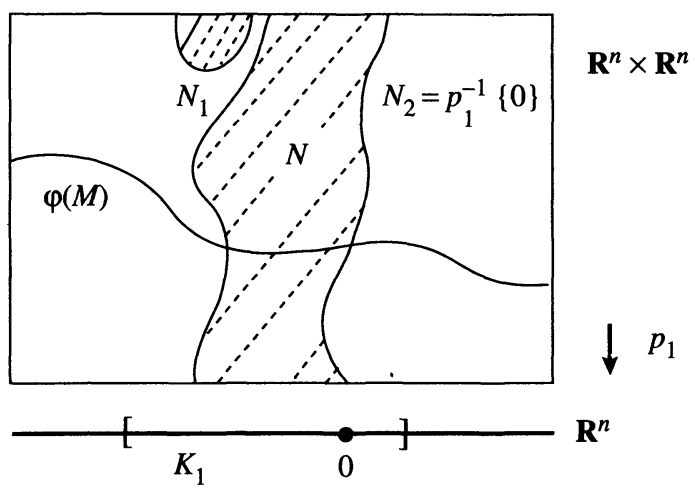

FIGURE 5

$\mathbf{R}^{n} \times \mathbf{R}^{n}$ surle premier facteur, et soit $\varphi$ une application continue de $M$ dans $\mathbf{R}^{n} \times \mathbf{R}^{n}, \psi$ une application continue de $N$ dans $\mathbf{R}^{n} \times \mathbf{R}^{n}$ telles que

(i) $p_{1} \circ \varphi$ est propre et de degré 1 .

(ii) $\psi$ est propre et $p_{1} \circ \psi$ est inclus dans un compact $K_{1}$.

(iii) $\psi$ est un homéomorphisme de $N_{2}$ dans $\{0\} \times \mathbf{R}^{n}$.

Alors $\psi\left(N_{2}\right)$ et $\varphi(M)$ s'intersectent.

Démonstration (voir Figure 5). La démonstration est élémentaire et est laissée au lecteur.

\section{Bibliographie}

[1] W. Ballman, M. Gromov \& V. Schroeder, Manifolds of non-positive curvature, Birkhäuser studies, Boston, 1985.

[2] R. D. Canary, D. B. A. Epstein \& P. Green, Notes on notes of Thurston, Analytical and Geometric Aspects of Hyperbolic Spaces, (D. B. A. Epstein, ed.), London Math. Soc. Lecture Notes Ser., No. 111, Cambridge University Press, Cambridge, 1987, 3-92.

[3] W. M. Goldman, Geometric structures on manifolds and varieties of representations, Contemporary Math., vol. 74, 1988, 169-198.

[4] M. Gromov, Partial differential relations, Springer, Berlin, 1986.

[5] F. Labourie, Problème de Minkowski et surfaces à courbures constantes dans les variétés hyperboliques, Bull. Soc. Math. France 119 (1991) 307-325.

[6] __ Surfaces convexes dans l'espace hyperboliques et $\mathbf{C P}^{1}$-structures, J. London Math. Soc., à paraître.

[7] __ Immersions isométriques elliptiques et courbes pseudo-holomorphes, J. Differential Geometry 30 (1989) 395-424.

[8] J. W. Morgan, Uniformization theorem for 3-dimensional manifolds, Chap. 5, Proc. Smith Conjecture Symposium (J. W. Morgan and H. Bass, eds.), Academic Press, New York, 1984. 
[9] A. V. Pogorelov, Extrinsic geometry of convex surfaces, Israel Program for Scientific Translation, Jerusalem, 1973.

[10] M. Spivak, A comprehensive introduction to differential geometry, Publish or Perish, Boston, MA, 1975.

[11] W. P. Thurston, Geometry and topology of 3-manifolds, Chap. 8, mimeographic notes.

Ecole Polytechnique

Palaiseau, France 